\title{
Pregnancy and isotretinoin therapy
}

\author{
June Seek Choi MD, Gideon Koren MD, Irena Nulman MD
}

A 29-year-old woman was given isotretinoin for severe, recalcitrant acne. During the first 3 months of treatment, she was taking an oral contraceptive agent that she had started at the same time as the isotretinoin. After deciding she wanted to become pregnant, the patient stopped taking the contraceptive agent but continued to take isotretinoin for another 2 months.

When the patient became pregnant, she sought advice from the Motherisk program regarding the fetal risks of exposure to isotretinoin. With counselling, it became apparent that the patient had not understood the connection between isotretinoin and birth defects, and hence the need for continued effective contraception while taking the drug. After repeated counselling, the patient decided to terminate her pregnancy.

\section{Discussion}

Oral isotretinoin was introduced in the United States in 1982 and in Canada in 1983 for the treatment of severe recalcitrant acne. In the intervening decades, additional off-label indications have become established, including the treatment of gram-negative folliculitis, recalcitrant rosacea, pyoderma facial, generalized lichen planus, psoriasis, cutaneous lupus erythematosus and acne fulminans.

Although effective for treating severe acne, isotretinoin is a potent human teratogen. There is an estimated $20 \%-35 \%$ risk for congenital defects in infants exposed to the drug in utero, ${ }^{1}$ including craniofacial, cardiovascular, neurological and thymic malformations. About 30\%-60\% of children exposed to isotretinoin prenatally have been reported to show neurocognitive impairment, even in the absence of physical defects. ${ }^{1}$

The gold standard for preventing fetal exposure to isotretinoin relies on the following tenets: the woman's awareness of the risks; ruling out pregnancy at the start of treatment; the use of 2 forms of contraception in parallel during treatment; monitoring for pregnancy throughout treatment; and avoiding pregnancy until the drug has been cleared from the body. ${ }^{2}$ Although these principles have been incorporated in all regulatory programs aimed at preventing fetal risk, repeated studies have shown failures in each step.

\section{Causes of isotretinoin exposure in pregnancy}

The most commonly reported reasons for pregnancy during isotretinoin therapy are unsuccessful attempts at abstaining from sexual intercourse, ineffective or inconsistent use of contraceptive agents or failure to detect pregnancy. ${ }^{2}$

Contraceptive failure is a leading reason for pregnancy during isotretinoin treatment. Despite acknowledging their familiarity with the risks of isotretinoin to the fetus, $7 \%-59 \%$ of women taking the drug do not use any form of contraception, ${ }^{3-5}$ and as many as $80 \%$ of women do not use 2 methods of birth control in parallel, as recommended. ${ }^{2.5}$ Our patient, for example, used only a single method of contraception. Improper use of contraceptives, in our experience, is the most common reason for unexpected pregnancies among women using isotretinoin and highlights the need for more comprehensive contraceptive counselling for all women starting treatment with this drug. ${ }^{2}$ Although most women know that taking isotretinoin confers a high risk of congenital defects if pregnancy occurs, ${ }^{3-5}$ they still choose to use it for the treatment of acne.

Many women taking isotretinoin do not monitor for pregnancy before and during treatment. Only $44 \%-67 \%$ of women report taking a preg-

\section{- Ker POINTS}

- Isotretinoin is a known teratogen, but women taking this drug may fail to use effective contraception.

- Current programs in Canada do not appear to be effective in preventing pregnancies during isotretinoin therapy.

- Prescribing physicians should ensure that women of reproductive age who take isotretinoin understand the fetal risks of this drug and use effective contraception.

- Preventing fetal exposure to isotretinoin will require system-level internventions in addition to those at the level of individual health care providers and patients.
Competing interests: None declared.

This article has been peer reviewed.

Correspondence to: Irena Nulman, irena.nulman@sickkids.ca

CMAJ 2013. DOI:10.1503 /cmaj.120729 
nancy test before starting isotretinoin therapy, and only about 1 in 5 reports taking a repeated pregnancy test. ${ }^{6}$ Moreover, most women use over-thecounter pregnancy tests for human chorionic gonadotropic hormone. These tests have limited sensitivity and may result in false-negative results, particularly if they are done shortly after conception. ${ }^{7}$ Repeat testing may lead to earlier detection of pregnancy and subsequent earlier cessation of treatment or pregnancy termination. ${ }^{8}$

Variability in the pharmacokinetics of isotretinoin may also lead to exposure during pregnancy. Continued use of contraception for 1 month after the end of isotretinoin therapy has been advocated to provide an adequate safety margin to prevent fetal exposure, based on more than 5 elimination half-lives of the drug. ${ }^{9}$ However, there is evidence of greater variability in isotretinoin elimination half-life (from $5.3 \mathrm{~h}$ to $7 \mathrm{~d}$ ); thus, 1 month may not allow clearing of the drug in all women..$^{10}$ Indeed, there have been reports of isotretinoininduced fetal malformations despite stopping treatment 1 month before pregnancy. ${ }^{11}$ A 3-month window may be more appropriate.

\section{Preventing fetal exposure to isotretinoin}

In the past 3 decades, several programs have been started in an attempt to prevent pregnancy during isotretinoin therapy. Although these programs have been successful in keeping pregnancy rates low relative to the general population of women of childbearing age who use contraception, they have all been ineffective in completely eliminating and preventing fetal exposure to the drug. In 1988, Roche and the US Food and Drug Administration (FDA) introduced the Retinoid Pregnancy Prevention Program. This program included explaining to women the risk to their unborn babies and obtaining their written agreement to use 2 methods of contraception in parallel, have monthly pregnancy tests and otherwise comply with the program. This program has been practised in Canada ever since. An evaluation of the program in 1995 showed that despite $99 \%$ of patients recalling being told to avoid pregnancy, $36 \%$ failed to receive a pregnancy test before starting treatment. ${ }^{12}$ An assessment in 2000 concluded that the program was unable to adequately prevent pregnancies during treatment, suggesting the need for improvement. ${ }^{13}$

In April 2002, the System to Manage Accutane-Related Teratogenicity (SMART) program began in the US to prevent women from starting isotretinoin therapy during pregnancy and to prevent conception during treatment. In addition to the steps included in the Retinoid Pregnancy Prevention Program, SMART called for 2 consecutive pregnancy tests with negative results before starting treatment and a voluntary registration system. The program did not include verification of compliance by physicians and pharmacists. Similar to the Retinoid Pregnancy Prevention Program, SMART has also been shown to be ineffective: $34 \%$ of women did not receive the required 2 pregnancy tests before starting treatment, and $54 \%$ of women of childbearing age did not use 2 methods of birth control in parallel during treatment. ${ }^{13}$

In 2006, the FDA implemented iPLEDGE, a program designed to provide more exhaustive guidelines for the safe prescription and use of isotretinoin. This program enforces monthly pregnancy tests, documentation of contraceptive use and patient education to reinforce key safety messages. Furthermore, all patients with prescriptions for isotretinoin and their pharmacists are registered in a single database. ${ }^{8}$ Seven years after its introduction, this program does not appear to have solved the issue of fetal exposure to isotretinoin. Shin and colleagues recently reported 29 fetal exposures out of a total of 9912 courses of treatment with the drug..$^{14}$ The authors found no evidence that iPLEDGE significantly decreased the risk of fetal exposure compared with the SMART program. Health Canada's Scientific Advisory Panel on isotretinoin has unanimously rejected the idea of a similar registry in Canada. ${ }^{15}$

In addition, we are facing a serious new challenge. After decades of the availability of only a single isotretinoin product (Accutane, Roche Inc.), there is now broad availability of multiple products, particularly on the Web. There are 232 types of generic and original isotretinoin made in 36 countries around the world, with 4 versions (Accutane, Accutane Roche, Clarus and Isotrex) currently available in Canada. Isotretinoin can be purchased online from different sources, without prescription or patient education. The amount of isotretinoin circulating in Canada is probably higher than estimated, and many users will not have received proper drug safety education.

\section{Multilevel approaches}

Preventing fetal exposure to isotretinoin requires intervention at multiple levels. In addition to the programs currently in place, other options for system intervention include banning online sales of isotretinoin, as well as mandatory online certification programs for the physicians and pharmacists providing the drug that include education about contraceptive methods and the drug's therapeutic and fetotoxic effects. A similar program currently exists for health care providers prescribing methadone in Canada, which appears to function well ${ }^{16}$

It is the responsibility of the prescribing physi- 
Box 1: Failure rate (\%) of common contraceptive methods under typical use

- Hormonal implant $=0.05$

- Combined injectable hormones $=0.2$

- Intrauterine device (IUD) with progesterone $=0.2$

- IUD (copper) $=0.8$

- Medroxyprogesterone acetate $=3$

- Spermicidal agents $=8$

- Combined contraceptive pill =8

- Male latex condom = 15

- Diaphragm and spermicide $=16$

- Standard day method (calendar method) $=25$

Source: General Practice Notebook, available at www gpnotebook.co.uk/simplepage.cfm?ID=-1590362086

cian to counsel women and their sexual partners as to the teratogenic effects of isotretinoin. This advice should include appropriate information about contraceptive methods and how to properly perform pregnancy tests. However, in a survey of internists, only $65 \%$ of responding physicians appeared to have correct knowledge of published failure rates of contraceptive agents (Box 1). ${ }^{17} \mathrm{In}$ that study, most of the physicians agreed there was a need for continuing medical education on counselling patients about potential teratogens and contraception. ${ }^{17}$ This education should precede patient education. ${ }^{17}$

Physicians in clinical practice are typically very busy, with little time to educate patients about effective contraception; some patients may have limited ability to understand written or verbal instructions in English or French; and teenage patients may be hesitant to discuss their sexual activities with health care providers. Appropriate contraceptive counselling takes time and may not be effectively achieved during an interview lasting only a few minutes. Multidisciplinary involvement may be helpful, including the reinforcement of information (both written and verbal) by pharmacists and other health care providers.

\section{Conclusion}

The medical community has a major role to play in ensuring that babies are not affected by prescription drugs taken during pregnancy. The continued occurrence of fetal exposure to isotretinoin highlights the need for improved practices. These improvements will likely require intervention at multiple levels, including improved education for patients and health care providers, improved patient adherence with established safety protocols and proper control of isotretinoin's distribution.

\section{References}

1. Lammer EJ, Chen DT, Hoar RM, et al. Retinoic acid embryopathy. N Engl J Med 1985;313:837-41.

2. Pastuszak A, Koren G, Rieder MJ. Use of the Retinoid Pregnancy Prevention Program in Canada: patterns of contraception use in women treated with isotretinoin and etretinate. Reprod Toxicol 1994;8:63-8.

3. Boucher N, Beaulac-Baillargeon L. Pregnancy prevention among women taking isotretinoin: failure to comply with the recommendations. Can Fam Physician 2006;52:338-9.

4. Garcia-Bournissen F, Tsur L, Goldstein LH, et al. Fetal exposure to isotretinoin - an international problem. Reprod Toxicol 2008;25:124-8

5. Honein MA, Paulozzi LJ, Erickson JD. Continued occurrence of Accutane-exposed pregnancies. Teratology 2001;64:142-7.

6. Robertson J, Polifka JE, Avner M, et al. A survey of pregnant women using isotretinoin. Birth Defects Res A Clin Mol Teratol 2005;73:881-7.

7. Cole LA, Ladner DG. Background hCG in non-pregnant individuals: need for more sensitive point-of-care and over-thecounter pregnancy tests. Clin Biochem 2009;42:168-75.

8. Abroms L, Maibach E. LyonDaniel Kl. What is the best approach to reducing birth defects associated with isotretinoin? PLoS Med 2006;3:e483.

9. Wiegand UW, Chou RC. Pharmacokinetics of oral isotretinoin. $J$ Am Acad Dermatol 1998;39:S8-12.

10. Nulman I, Berkovitch M, Klein J, et al. Steady-state pharmacokinetics of isotretinoin and its 4-oxo metabolite: implications for fetal safety. J Clin Pharmacol 1998;38:926-30.

11. Lee SM, Kim HM, Lee JS, et al. A case of suspected isotretinoin-induced malformation in a baby of a mother who became pregnant one month after discontinuation of the drug. Yonsei Med J 2009;50:445-7.

12. Mitchell AA, Van Bennekom CM, Louik C. A pregnancyprevention program in women of childbearing age receiving isotretinoin. N Engl J Med 1995;333:101-6.

13. Brinker A, Kornegay C, Nourjah P. Trends in adherence to a revised risk-management program designed to decrease or eliminate isotretinoin-exposed pregnancies: evaluation of the Accutane SMART program. Arch Dermatol 2005;141:563-9.

14. Shin J, Cheetham TC, Wong L, et al. The impact of the iPLEDGE program on isotretinoin fetal exposure in an integrated health care system. J Am Acad Dermatol 2011;65:1117-25.

15. Andresen M. Accutane registry compulsory in US, but not Canada. CMAJ 2006;174:1701.

16. Fischer B. Prescriptions, power and politics: the turbulent history of methadone maintenance in Canada. J Public Health Policy 2000;21:187-210.

17. Eisenberg DL, Stika C, Desai A, et al. Providing contraception for women taking potentially teratogenic medications: a survey of internal medicine physicians' knowledge, attitudes and barriers. J Gen Intern Med 2010;25:291-7.

Affiliations: From the Motherisk Program (Choi, Koren, Nulman) Division of Clinical Pharmacology/Toxicology, Hospital for Sick Children, Toronto, Ont., and Cheil General Hospital and Women's Healthcare Centre (Choi), Seoul, Republic of Korea.

Contributors: June Seek Choi wrote the first draft of the paper, and revised and edited it for important intellectual content. Irena Nulman participated in conception and design and the paper, and the acquisition and interpretation of the data. Gideon Koren participated in the concept and design of the paper, its drafting and revision, and the interpretation of the data. All of the authors approved the final version submitted for publication. 